\title{
Effectiveness of ultrasound-guided percutaneus neuromodulation on peripheal neuropathies induced by chemotherapy: a pilot study
}

\author{
López-Garzón M.C. ${ }^{1}$ Lozano-Lozano M. ${ }^{1}$ Álvarez-Salvago F. ${ }^{1}$ Galiano-Castillo N. ${ }^{1}$ \\ ${ }^{1}$ Department of Physiotherapy. Faculty of Health Sciences, \\ Universidad de Granada, Granada, Spain \\ Rev Fisioter Invasiva 2019;2:84-85.
}

\begin{abstract}
Keywords

- neoplasia

- chemotherapy

- chemotherapy induced peripheral neuropathy

- pain
\end{abstract}

Background and Aims Chemotherapy-induced peripheral neuropathy (CIPN) is one of the most invalidating secondary effects (60\%) both of colorectal cancer as well as breast cancer and is a reason for abandoning medical treatment. The symptoms are based on pain, tingling, numbness, and can affect quality of sleep. It is known that analgesia induced by TENS is multifactorial and probably comprises peripheral, spinal and supraspinal mechanisms. Besides, these effects could be greater if the application is performed percutaneously. Therefore, the principal aim of this study was to evaluate the effectiveness of ultrasound-guide percutaneous neuromodulation on the quality of life of patients who suffer CIPN.

Material and Methods Three patients $(n=2$ breast cancer and $n=1$ colorectal cancer) were recruited from the University Hospital Virgen de las Nieves (Granada, Spain). This study received ethics committee approval (0110-N-18) The radial, cubital and median nerves were treated in the upper limb, and the tibial, peroneal, sural and saphenous nerves were treated for the lower limb. In total, eight treatment sessions were performed per affected limb, using the treatment protocol of $\mathrm{NMP}^{\circledR}$ (20 seconds of TENS $10 \mathrm{~Hz} 2 \mathrm{~mA}$ and 10 minutes of TENS $2 \mathrm{~Hz} 1-10 \mathrm{~mA}$ and in the sural nerve. Besides, 5 minutes of TENS was applied TENS $50 \mathrm{~Hz} 1-10 \mathrm{~mA}$ ) in a weekly session during two months. Before and after treatment, the following variables were evaluated: 1) neuropathy specific quality of life (EORTC QLQ-CIPN20) consisting of three subscales (0-100): sensory, autonomic, motor (greater conduction); 2) intensity of symptoms (numbness, pain and tingling) using the visual analog scale (VAS) (0-10); and 3) sleep according to the Pittsburgh Sleep Quality Index (0-21) using its total component. For the analyses, the normality of the population was assumed due to the nature of the study $(\mathrm{N}=3)$ and therefore the Student's t-test was used to analyze the differences in the baseline and post-intervention means using the IBM SPSS Statistics 21 software (IBM Corporation, Armonk, NY).

Results Regarding the autonomic subscale (baseline mean, SD $38.89 \pm 5.56$ ) of the EORTC QLQ-CIPN20 the difference of means was statistically significant $(-38.89 \pm 9.62)$ $(t=-6.99 ; P=0.020)$. The remaining subscales were not significant $(P>0.05)$ despite the fact that the results also improved: sensory $(-2.47+-16.7)$; motor $(-26.89 \pm 19.25)$; and conduction $(-11.11 \pm 19.25)$. The VAS for pain (baseline mean, SD: $5 \pm 2.52$ ) obtained a difference in means of $-1.67 \pm 2.08$, although it was not significant
DOI https://doi.org/ $10.1055 / \mathrm{s}-0039-3401866$. ISSN 2386-4591.
Copyright $\odot 2019$ by Thieme Revinter Publicações Ltda, Rio de Janeiro, Brazil
License terms

$\circledast(1) \Theta \circledast$ 
$(P>0.05)$ it did reach a clinically relevant difference (1.1 points). For the remaining VAS scales, numbing and tingling, no statistically significant differences were obtained $-1.17 \pm 2.021$ and $-1.5 \pm 1.80$, respectively $(P>0.05)$. Lastly, the Pittsburgh questionnaire (baseline mean: 10 ) decreased by $-3.67 \pm 1.53$ showing a trend towards significance $(t=-4.19 ; P=0,053)$.

Conclusion Despite this being a pilot study, percutaneous neuromodulation is shown to improve the symptoms of neuropathy induced by chemotherapy, specifically autonomic symptoms and quality of sleep. Our results may represent a change of paradigm in physical therapy treatment. 